\title{
Posterior interosseous nerve discontinuity due to compression by lipoma: report of 2 cases
}

\author{
Andrés A. Maldonado, MD, PhD, Benjamin M. Howe, MD, and Robert J. Spinner, MD \\ Departments of Neurologic Surgery and Radiology, Mayo Clinic, Rochester, Minnesota
}

Paralysis of the posterior interosseous nerve (PIN) secondary to compression is a rare clinical condition. Entrapment neuropathy may occur at fibrous bands at the proximal, middle, or distal edge of the supinator. Tumors are a relatively rare but well-known potential cause. The authors present 2 cases of PIN lesions in which compression by a benign lipoma at the level of the elbow resulted in near transection (discontinuity) of the nerve. They hypothesize a mechanisma "sandwich effect"-by which compression was produced from below by the mass and from above by a fibrous band in the supinator muscle (i.e., the leading edge of the proximal supinator muscle [arcade of Fröhse] in one patient and the distal edge of the supinator muscle in the other). A Grade $V$ Sunderland nerve lesion resulted from the advanced, chronic compression. The authors are unaware of a similar case with such an advanced pathoanatomical finding.

https://thejns.org/doi/abs/10.3171/2016.2.JNS152810

KEY WORDS posterior interosseous nerve; radial nerve; arcade of Fröhse; supinator; compression; paralysis; palsy; peripheral nerve

$\mathrm{C}$ OMPRESSION of the posterior interosseous nerve (PIN) is a relatively rare but well-known disorder. Entrapment may occur spontaneously at points of potential vulnerability related to the proximal, middle, or distal supinator muscle. ${ }^{3,410,16,21}$ Mass lesions, particularly lipomas, are well-described causes of secondary compression. ${ }^{1}$

We present 2 cases in which a lipoma in the elbow region resulted in PIN compression and nerve discontinuity. A Grade V nerve injury according to the Sunderland classification was found in both cases..$^{19}$ We believe that this occurs due to a chronic compression mechanism: a combination of forces act on the nerve, including the mass itself and a point of potential entrapment ("sandwich effect"). In these 2 cases compression occurred from below by the lipoma and from above by a fibrous band within the supinator muscle (the leading edge of the proximal supinator muscle [arcade of Fröhse] in one patient and the distal edge of the supinator muscle in the other). We are unaware of a previous description of this pathology.

\section{Case Reports}

Two patients (a 78-year-old man [Case 1] and a 65-yearold woman [Case 2]) presented with an 8-month and a 2.5-year history, respectively, of an enlarging mass in the right proximal forearm. The first patient noted difficulty extending the right little and ring fingers initially, and then all fingers and the wrist. The second patient became aware of weakness of her right index finger, which then progressed to the others. Neither had pain, although each described a subtle ache in the forearm.

Both patients were evaluated multiple times at different medical centers, but without a clear description of the muscle grading of the finger extensors or a definitive diagnosis. Physical examination at our institution revealed evidence of a complete PIN paralysis manifested by wrist dorsiflexion in a radial deviation and finger drop. Sensation was normal. Electrophysiology studies confirmed a complete right PIN neuropathy. Imaging (ultrasound in 1 case [Fig. 1A] and MRI in both cases [Figs. 1B, 1C, and $2 \mathrm{~A}-\mathrm{F}]$ ) demonstrated a $4.5 \times 5.2-\mathrm{cm}$ (Case 1 ) and $4 \times 6.5-$ $\mathrm{cm}$ (Case 2) lipoma adjacent to the neck of the proximal radius displacing the PIN.

An anterior (Henry) approach to the proximal forearm was performed in both patients. Proximal and distal control of the superficial and deep branches of the radial nerve was obtained. In each case, during the mobilization of the lipoma (Figs. 1D, 1E, 2G, and 2H), severe compression of the PIN was evident directly beneath the supinator muscle. The PIN was indented and appeared in discontinuity, with only a few viable fascicles remaining (Figs. 1E and 2I). In Case 1 , the parosteal lipoma was largely within the supinator and extended superiorly to a lesser degree. The compression was localized to the proximal edge of the supinator muscle (arcade of Fröhse). In Case 2, the lipoma was compressing the nerve at the level of the distal edge of the supinator. 

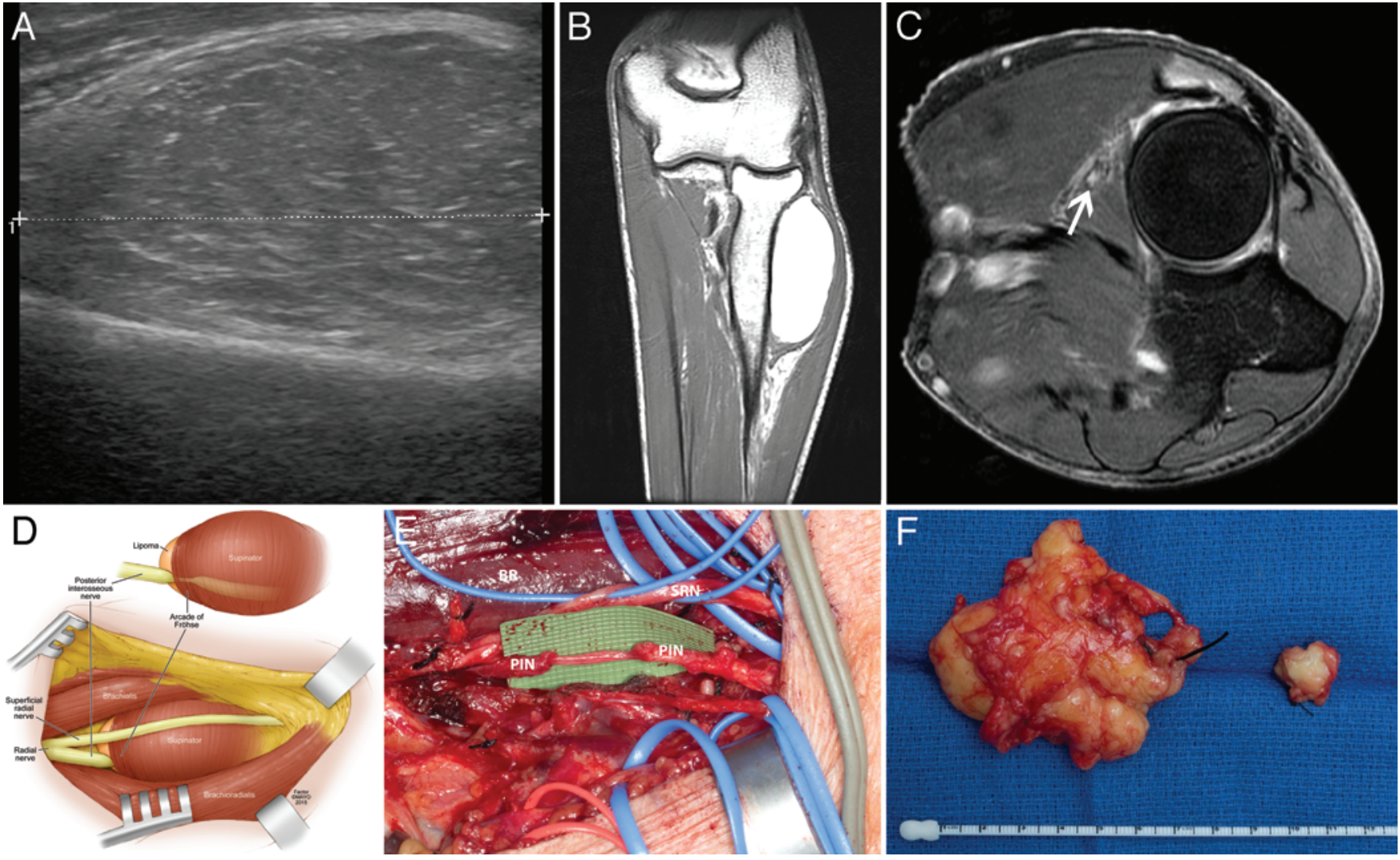

FIG. 1. Case 1. A: Ultrasound examination revealing a $5-\mathrm{cm}$, spindle-shaped heterogeneous mass. B: Coronal T1-weighted image of the right elbow and proximal forearm demonstrating a large lipoma extending from the radial head/neck junction to the proximal radial shaft. C: Axial T2-weighted fat-saturated image demonstrating enlargement and hyperintensity of the deep branch of the radial nerve (arrow) just proximal to the arcade of Fröhse, proximal to the lipoma at the level of the radial head. D: Sandwich effect schematic illustration of the right proximal forearm. Note the compression of the PIN between the lipoma and the arcade of Fröhse. Used with permission of the Mayo Foundation for Medical Education and Research. All rights reserved. E: Intraoperative photograph showing the right PIN lesion after dissection. F: Macroscopic view of the tumor after resection. BR = brachialis muscle; SRN = superficial radial nerve.

The patients' tumors were resected. Postoperatively, because of the poor prognosis for spontaneous recovery, both were recommended to undergo standard tendon transfers: the first patient (Case 1) received them at 3 months with good results, whereas the second patient (Case 2) wished to undergo the same procedure at 6 months.

In both instances, after the surgery we reinterpreted the MR images. Previously, only PIN displacement and hyperintensity had been detected on formal review. Now, compression of the PIN at the arcade of Fröhse in the first patient (Fig. 1C) and at the proximal and distal margin of the supinator in the second patient (Fig. 2B and 2D) could be observed and correlated. In this second patient, a large neuroma and then more distally a diminished-caliber nerve was seen in the PIN at the distal margin of the supinator (Fig. 2E).

\section{Discussion}

Paralysis of the PIN by a lipoma is rare but well described. A review in 2004 by Avram and Hynes ${ }^{1}$ revealed only 29 cases in the English literature. Several additional cases have been reported in the last 10 years. ${ }^{2,5-7,9,14,22}$ Typi- cally, after resection of lipomas, PIN recovery is anticipated. ${ }^{1}$ We present 2 patients in whom the compression led to near discontinuity of the nerve; given the severity of the lesion, spontaneous recovery would not be anticipated and tendon transfers were offered early. Nerve grafting or nerve transfers were not considered due to their anticipated poor results from the chronicity and severity of the PIN injury; instead tendon transfers were offered due to their predictably favorable outcomes.

We believe that the pathophysiology in these 2 cases was due to the presence of the lipoma at a potential source of nerve compression. Compression of the PIN was produced by the forces of the lipoma from below and the supinator muscle from above. As in a sandwich, 2 pieces of bread (supinator and lipoma) compress the cheese (PIN) in between (Figs. 1D and 2G). Interestingly, in Case 2, in which the nerve had a dumbbell configuration, there was compression at both the proximal and distal edges of the supinator. With time and/or growth of the mass, the blood supply to the nerve could be compromised, producing the advanced pathology (axonal and neural discontinuity).

Partial nerve section is an extreme finding in cases of nerve compression. Le Hanneur et al. ${ }^{11}$ reported partial 

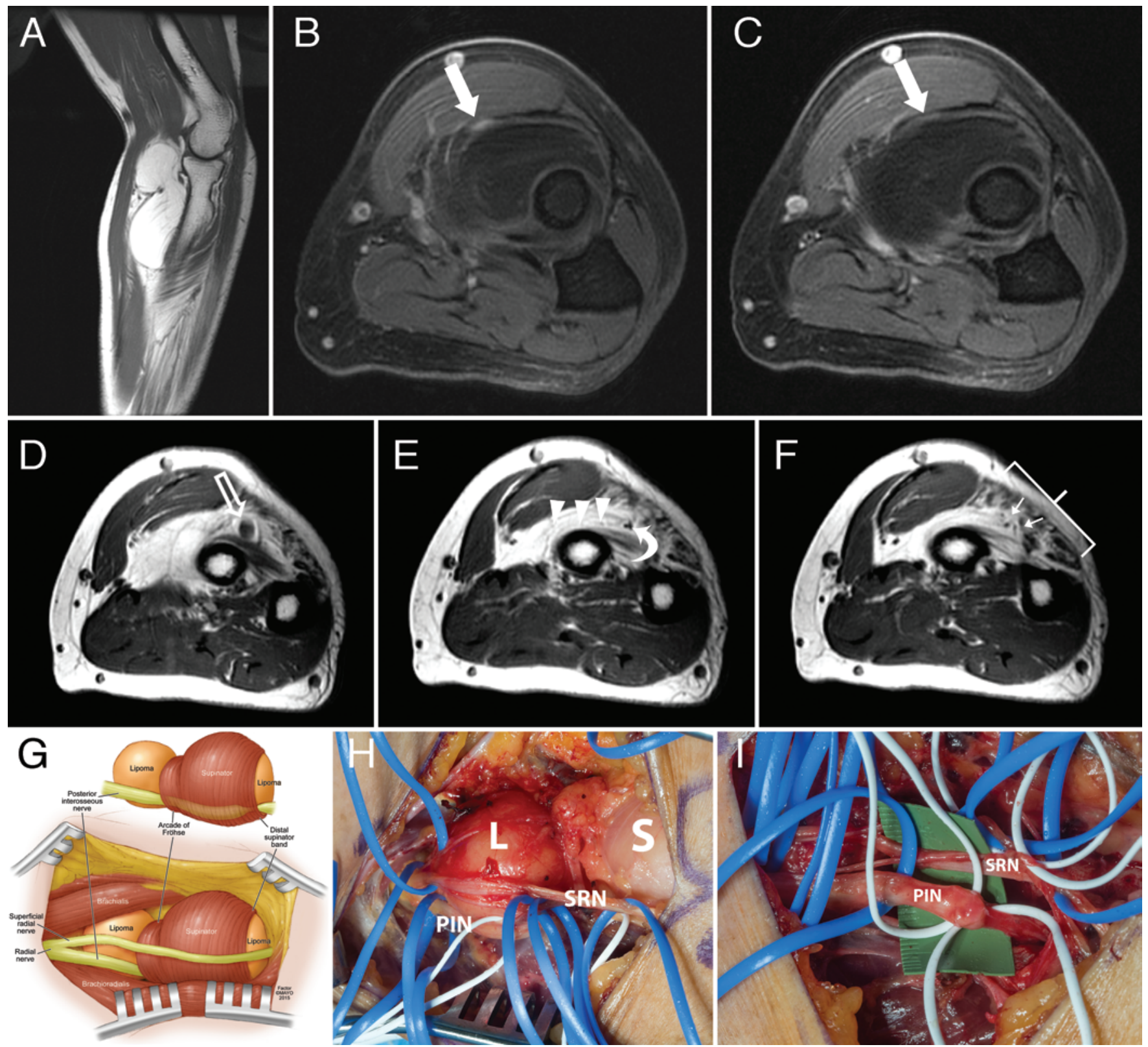

FIG. 2. Case 2. A: Sagittal T1-weighted image of the right elbow and proximal forearm showing a large lipoma that extends from the level of the elbow joint to the proximal radial shaft. B and C: Sequential axial T2-weighted fat-saturated images of the right proximal forearm demonstrating compression of the deep branch of the radial nerve (arrows) at the proximal margin of the supinator. B: Nerve enlargement and T2-weighted hyperintensity are revealed. C: One slice distal to panel B. Note the compression of the deep branch of the nerve at this level. D-F: Sequential axial T1-weighted images of the forearm demonstrating compression of the PIN at the distal margin of the supinator. D: A large neuroma is present in the PIN just prior to exiting the supinator (open arrow). E: Two slices distal to panel D. The PIN is decreased in caliber (curved arrow) at the distal margin of the supinator (arrowheads). F: Four slices distal to panel D. The PIN arborizes just distal to the supinator (arrows). Note the chronic denervation atrophy and fatty replacement of the extensor compartment of the forearm (bracket). G: Sandwich effect schematic illustration of the right proximal forearm. Note the compression of the PIN between the lipoma and the proximal and distal edges of the supinator. Used with permission of the Mayo Foundation for Medical Education and Research. All rights reserved. H: Intraoperative photograph showing the resection of the right proximal forearm tumor. Note the relationship with deep and superficial branches of the radial nerve. I: Intraoperative photograph showing the PIN. Note how the nerve is in almost total discontinuity, with a neuroma in the proximal portion. $\mathrm{L}=$ lipoma; $\mathrm{S}=$ supinator muscle.

section of the suprascapular nerve by the superior transverse scapular ligament. A second paper by Thoma et al..$^{20}$ described an hourglass deformity with a "near autoamputation" of the PIN at the level of the supinator muscle. It is unknown whether the operative findings in these cases are due to entrapment or another mechanism such as inflammation. Fascicular torsion leading to hourglass deformity of different nerves including the PIN has been described.,12 
The association of these deformities and inflammatory neuropathy, such as Parsonage-Turner syndrome, has been recently established. ${ }^{18,23}$ Nerve injury after chronic compression has been studied in animal models. ${ }^{13,15,17}$ The model with the longest period of compression is based on 6 months of sciatic nerve compression in rats. ${ }^{13}$ Macroscopically the nerve had an hourglass type shape but without nerve discontinuity. It is possible that nerve compression in these animal models was not long or severe enough to section the nerve as we found in our patients.

Our cases highlight 2 important points. First, the delay between the diagnosis of a PIN neuropathy and the lipoma resection was prolonged. We recommend that patients with PIN syndrome undergo imaging (either ultrasound or MRI). Second, these cases point out the susceptibility of the PIN while it traverses the supinator muscle, even by "soft" soft-tissue tumors (such as lipomas), creating a sandwich effect. We believe this mechanism may be applicable to other nerve lesions at other sites.

Our report has some limitations. First, we did not perform a histopathological investigation of the PIN injury. Only a small biopsy was taken from a portion of the neuroma in the second patient, and it showed standard chronic nerve injury. Second, the patients were not asked to give their consent for alternative reconstructive options because we did not expect to find such an advanced lesion involving the PIN. Tendon transfers, nerve grafts, and nerve transfers should be discussed with future patients prior to the tumor resection.

\section{Conclusions}

We describe partial PIN section by a benign lipoma. We hypothesize that the compression between the supinator muscle and the tumor compromised the blood supply of the PIN (sandwich effect), causing a Grade V Sunderland lesion. These cases highlight the importance of imaging, early diagnosis, and surgical management of PIN neuropathy, and the susceptibility of this nerve while it traverses the supinator muscle.

\section{References}

1. Avram R, Hynes NM: Posterior interosseous nerve compression secondary to a parosteal lipoma: Case report and literature review. Can J Plast Surg 12:69-72, 2004

2. Borman P, Tuncay F, Ulusoy G, Koçer U: Posterior interosseous nerve syndrome due to lipoma. Neurophysiol Clin 40:189-191, 2010

3. Clavert P, Lutz JC, Adam P, Wolfram-Gabel R, Liverneaux P, Kahn JL: Frohse's arcade is not the exclusive compression site of the radial nerve in its tunnel. Orthop Traumatol Surg Res 95:114-118, 2009

4. Derkash RS, Niebauer JJ: Entrapment of the posterior interosseous nerve by a fibrous band in the dorsal edge of the supinator muscle and erosion of a groove in the proximal radius. J Hand Surg Am 6:524-526, 1981

5. El Hyaoui H, Hassoun J, Garch A, Kassimi H, El Fatimi A: Compression of the posterior interosseous nerve by a deep lipoma. Joint Bone Spine 81:265, 2014

6. Eralp L, Ozger H, Ozkan K: [Posterior interosseous nerve palsy due to lipoma.] Acta Orthop Traumatol Turc 40:252-254, 2006 (Turkish)

7. Ganapathy K, Winston T, Seshadri V: Posterior interosse- ous nerve palsy due to intermuscular lipoma. Surg Neurol 65:495-496, 2006

8. Guerra WKW, Schroeder HWS: Peripheral nerve palsy by torsional nerve injury. Neurosurgery 68:1018-1024, 2011

9. Hamdi MF, Aloui I, Allagui M, Abid A: Paralysis of posterior interosseous nerve caused by parosteal lipoma. Neurol India 58:319-320, 2010

10. Knutsen EJ, Calfee RP: Uncommon upper extremity compression neuropathies. Hand Clin 29:443-453, 2013

11. Le Hanneur M, Teboul F, Goubier JN: Suprascapular nerve partial section by the transverse scapular ligament: one case. Plast Reconstr Surg Glob Open 3:e444, 2015

12. Loizides A, Baur EM, Plaikner M, Gruber H: Triple hourglass-like fascicular constriction of the posterior interosseous nerve: a rare cause of PIN syndrome. Arch Orthop Trauma Surg 135:635-637, 2015

13. Mackinnon SE, Dellon AL, Hudson AR, Hunter DA: Chronic nerve compression-an experimental model in the rat. Ann Plast Surg 13:112-120, 1984

14. Matsuo T, Sugita T, Shimose S, Kubo T, Yasunaga Y, Ochi M: Intraneural lipoma of the posterior interosseous nerve. J Hand Surg Am 32:1530-1532, 2007

15. Rempel DM, Diao E: Entrapment neuropathies: pathophysiology and pathogenesis. J Electromyogr Kinesiol 14:71-75, 2004

16. Riffaud L, Morandi X, Godey B, Brassier G, Guegan Y, Darnault $\mathrm{P}$, et al: Anatomic bases for the compression and neurolysis of the deep branch of the radial nerve in the radial tunnel. Surg Radiol Anat 21:229-233, 1999

17. Sommer C, Galbraith JA, Heckman HM, Myers RR: Pathology of experimental compression neuropathy producing hyperesthesia. J Neuropathol Exp Neurol 52:223-233, 1993

18. Sumner AJ: Idiopathic brachial neuritis. Neurosurgery 65 (4 Suppl):A150-A152, 2009

19. Sunderland $\mathrm{S}$ : A classification of peripheral nerve injuries producing loss of function. Brain 74:491-516, 1951

20. Thoma A, Ching S, Nelluri P: Outcomes of surgical treatment in posterior interosseous nerve syndrome. Can J Plast Surg 10:210-213, 2002

21. Tubbs RS, Mortazavi MM, Farrington WJ, Chern JJ, Shoja MM, Loukas M, et al: Relationships between the posterior interosseous nerve and the supinator muscle: application to peripheral nerve compression syndromes and nerve transfer procedures. J Neurol Surg A Cent Eur Neurosurg 74:290-293, 2013

22. Yamamoto D, Yamauchi D, Tsuchiya H: Intraneural lipoma of the posterior interosseous nerve. J Hand Surg Eur [epub ahead of print], 2015

23. Yongwei P, Guanglei T, Jianing W, Shuhuan W, Qingtai L, Wen T: Nontraumatic paralysis of the radial nerve with multiple constrictions. J Hand Surg Am 28:199-205, 2003

\section{Disclosures}

The authors report no conflict of interest concerning the materials or methods used in this study or the findings specified in this paper.

\section{Author Contributions}

Conception and design: Spinner. Acquisition of data: Maldonado. Analysis and interpretation of data: all authors. Drafting the article: Maldonado. Critically revising the article: all authors. Reviewed submitted version of manuscript: all authors. Approved the final version of the manuscript on behalf of all authors: Spinner.

\section{Correspondence}

Robert J. Spinner, Department of Neurologic Surgery, Mayo Clinic, 200 1st St. SW, Gonda 8-214, Rochester, MN 55905. email: spinner.robert@mayo.edu. 